\title{
On the determinants of induction in responding for sucrose when food pellet reinforcement is upcoming
}

\author{
JEFFREY N. WEATHERLY, KARYN M. PLUMM, and JULIA R. SMITH \\ University of North Dakota, Grand Forks, North Dakota \\ and \\ WILLIAM A. ROBERTS \\ University of Western Ontario, London, Ontario, Canada
}

\begin{abstract}
Rats' rates of leverpressingfor low-concentration liquid-sucrose reinforcers in the first half of an experimental session increase when food pellet, rather than sucrose, reinforcers will be available in the second half. Experiment 1 determined that this induction effect was the outcome of food pellet reinforcement's increasing response rates, not of continued sucrose reinforcement's decreasing them. Experiments 2 and 3 showed that induction was primarily controlled by the conditions of reinforcement in the current session, not by those in the previous one. Experiment 4 showed little evidence that the induction was the outcome of Pavlovian processes. These results suggest that induction may occur because of processes operating at the level of the entire session. They also provide a link to a seemingly related area of study: contrast effects. Some of the results are consistent with what is known about contrast effects, but there are also several, yet unexplained differences.
\end{abstract}

Behavioral contrast (Reynolds, 1961) can be defined as an inverse relationship between the rate of behavior in one situation-typically, one component of a multiple schedule of reinforcement - and the conditions of reinforcement in another (McSweeney \& Norman, 1979). Much research has been conducted to determine under what conditions contrast will occur (see McSweeney \& Weatherly, 1998). Beyond basic empirical findings, numerous theoretical accounts have been put forward in an attempt to explain when and why contrast occurs (see, e.g., Williams, 2002, for a review).

Theoretical explanations for contrast have generally fallen into three categories. The first attributes the occurrence of contrast to procedural variations between conditions (e.g., McSweeney \& Weatherly, 1998), the second attempts to identify general mechanisms that could lead to contrast (e.g., Hinson \& Staddon, 1978), and the third invokes cognitive processes (see Williams, 2002, for a discussion of each type of explanation). Yet contrast remains intriguing for several reasons. For one, it has, to date, defied adequate explanation. Second, its occurrence is counter-

Portions of these data were made possible through funds provided to J.N.W. from the Office of Research and Program Development at the University of North Dakota and from the North Dakota EPSCoR, a subprogram of the National Science Foundation. The authors thank Emily Arthur, Michael Himle, Brent King, Patricia Moulton, and Jasen Ritt for their help in collecting the data. Correspondence regarding this article should be addressed to J. N. Weatherly, Department of Psychology, University of North Dakota, Grand Forks, ND 58202-8380(e-mail: jeffrey_ weatherly@und.nodak.edu). intuitive. When a lean component (and stimuli associated with it) consistently alternates with a rich component, it would seem reasonable to expect responding in the lean component to increase, rather than decrease, because the component and its associated stimuli would become a conditioned reinforcer (or an excitatory conditioned stimulus). However, such an increase, which would be considered an induction or reinforcement effect, is only sometimes reported (Baron \& Derenne, 2000; Flaherty \& Grigson, 1988). When it is reported, it has typically occurred in procedures in which there is a response requirement in the lean component for entry into the rich one.

Recently, however, a series of studies have been reported that show a reliable induction-like effect (e.g., Weatherly, Davis, \& Melville, 2000; Weatherly, Stout, Rue, \& Melville, 2000). Rats' rates of leverpressing for lowconcentration sucrose reinforcers delivered by a random interval (RI) schedule in the first half of an experimental session have been shown to increase when food pellet, rather than the same sucrose, reinforcers will be available in the second half. In short, upcoming food pellet reinforcement, which can be considered an increase in the conditions of reinforcement relative to low concentrations of liquid sucrose, ${ }^{1}$ appears to facilitate (induction), rather than suppress (contrast), response rates maintained by sucrose reinforcement.

Empirical studies on this potential induction effect have provided some insight into why it may occur. The relative value of the reinforcers appears to mediate the effect. Weatherly, Stout, et al. (2000) found that upcoming food pellet reinforcement produced a large induction-like effect 
when subjects responded for $1 \%$ liquid sucrose but produced little or no effect when they responded for $25 \%$ sucrose. Also, the likelihood that food pellets will be available influences the increase in responding. Weatherly, Davis, and Melville (2000) varied, across conditions, the probability that food pellet reinforcers would be available in the second half of a session and found that the increases in response rates across the first half of the session varied directly with the probability that food pellet reinforcement would be upcoming.

Several attempts have also been made to identify the theoretical underpinnings of the effect. For example, subjects may increase their response rate for sucrose when food pellet reinforcement is upcoming, because they are readying themselves to respond at a high rate for the food pellets. However, Weatherly, Moulton, and Ritt (2002) found that upcoming food pellets increased operant response rates for $1 \%$ sucrose even when the pellets would be delivered independently of responding.

Another potential explanation is that subjects are emitting "anticipatory" responses for the upcoming food pellets prior to their availability. To test this idea, Weatherly, Himle, Plumm, and Moulton (2001, Experiment 3) had rats respond for $1 \%$ sucrose and food pellet reinforcement in the first and second halves, respectively, of a session. The subjects responded on different levers in each half. "Anticipatory" responses did occur in the first half of the session on the lever that would deliver food pellets in the second half. However, response rates on the lever that delivered sucrose in the first half of the session were still increased. Thus, subjects may emit responses for the yetunavailable food pellets, but the addition of these responses cannot account for the apparent induction.

In the present study, the determinants of this supposed induction effect were investigated. Such an investigation seemed warranted for several reasons. First, although its occurrence may be more intuitive than a matter of contrast, the reasons for its occurrence remain unknown. Second, because the procedure in which it is observed shares similarities with procedures that produce contrast, understanding why it occurs has the potential to shed light on the phenomenon of contrast. For example, if the inductionlike increase in responding occurs through Pavlovian processes, support would be provided for the view that contrast occurs despite, not because of, Pavlovian influences (Williams \& McDevitt, 2001; and see Williams, 2002).

Experiment 1 tested the assumption that the increase in responding for sucrose occurred because of the availability of food pellet reinforcement. Previous studies (Weatherly, Davis, \& Melville, 2000; Weatherly, Himle, et al., 2001; Weatherly et al., 2002; Weatherly, Stout, et al., 2000) have reported that response rates for sucrose increased when food pellet, rather than the same sucrose, reinforcement was available in the second half of the session. However, it is also possible that continued sucrose reinforcement actually decreases response rates. Determining which possibility is true would help link induction to other phenomena (e.g., incentive contrast effects; see Flaherty, 1996, for a review) that occur because of differ- ential reinforcement in another situation. If the supposed induction occurs because continued sucrose reinforcement suppresses responding, the potential theoretical overlap between the two effects would be diminished.

Experiment 2 determined whether the induction is linked to the upcoming conditions of reinforcement. Although it is possible that the increase in responding for sucrose occurs because of prospective factors (i.e., food pellets will be available later in the session), it is also possible that it occurs because of retrospective ones (i.e., food pellets were obtained in the previous session). Williams (1983, 2002) has argued that steady-state behavioral contrast is largely, if not completely, controlled by prospective factors. Thus, if induction is also prospective, it is possible that contrast or induction occurs because of deviations in the same causal mechanism. If induction is retrospective, one could conclude that the factors leading to contrast or induction are independent.

Experiment 3 systematically replicated Experiment 2. It also determined whether certain stimuli present within the experimental context controlled the presence of induction. Establishing that such stimulus control occurred was important because previous studies on the relative effects of differential reinforcement have suggested that different causal mechanisms may be at work when such stimulus control is present (e.g., Flaherty \& Rowan, 1985) versus when it is absent (e.g., Timberlake \& Engle, 1995). If stimulus control can be established, attention can be focused on understanding one causal mechanism, rather than on trying to delineate between competing mechanisms.

In Experiment 4, the question of whether the stimulus control in Experiment 3 was the outcome of Pavlovian processes was investigated. Making this determination was again important for linking induction to other areas of study. Pavlovian conditioning has long been considered to play a role in the appearance of contrast (e.g., Rachlin, 1973). Recently, however, some researchers (Williams, 2002; Williams \& McDevitt, 2001) have argued that Pavlovian influences actually compete against its appearance. By identifying whether Pavlovian processes underlie induction, it is potentially possible to relate the causal factors of induction to those of contrast.

Finally, in each experiment, separate groups were employed that responded for either $1 \%$ or $5 \%$ sucrose reinforcement. Different sucrose concentrations were studied for three reasons. First, doing so allowed for a measure of reliability within each experiment. Second, the size of induction-like effects has been reported to vary inversely with sucrose concentration (e.g., Weatherly, Stout, et al., 2000). Thus, the present study provided an opportunity to replicate this result, as well as to determine whether there would be an interaction between sucrose concentration and any of the independent variables manipulated in the present experiments. Third, having subjects respond for different sucrose concentrations was potentially relevant to the predictions in the present experiments. For instance, Experiment 1 tested whether upcoming sucrose reinforcement decreased response rates in the first half of the ses- 
sion, rather than upcoming food pellet reinforcement increasing them. If such were the case, one might expect different levels of suppression by the different sucrose concentrations.

\section{EXPERIMENT 1}

Although previous studies (e.g., Weatherly, Davis, \& Melville, 2000; Weatherly, Rue, et al., 2000; Weatherly, Stout, et al., 2000) have reported that upcoming food pellet reinforcement increases rats' rates of responding for liquid sucrose reinforcement, it remains possible that sessions in which sucrose reinforcement will be available in both halves of the session actually produce lower rates of responding in the first half than would sessions in which no reinforcement is available in the second half (or in which there is no second half). In short, it is possible that response rates when food pellets are upcoming actually represent the "normal" state of affairs (i.e., the absence of suppression).

The subjects in Experiment 1 responded in four conditions. In one, sucrose reinforcement was available throughout the 50-min session. In another, sucrose reinforcement was available in the first half of the session, and food pellet reinforcement was available in the second half. In a third, the subjects responded for sucrose in the first half of the session, and extinction was in effect in the second half. In the final condition, the subjects responded for sucrose in a 25-min session (i.e., there was no second half). If upcoming sucrose reinforcement suppresses current responding, then response rates in the first half of the session should be lower when sucrose reinforcement will be available in the second half of the session than when other conditions of reinforcement (e.g., extinction) exist in the second half.

By having different groups respond for either $1 \%$ or $5 \%$ sucrose, it was also potentially possible to identify the mechanism by which sucrose reinforcement in the second half of the session might suppress responding. If the potential suppression occurs because of the low value of the sucrose available in the second half of the session, one would predict the greatest suppression for subjects responding for $1 \%$ sucrose. If, on the other hand, the potential suppression occurs because of the high value of the sucrose in the second half of the session (e.g., inhibition by reinforcement; e.g., Catania, 1973), one would predict the greatest suppression for subjects responding for $5 \%$ sucrose.

\section{Method}

Subjects. The subjects were 12 experimentally experienced male Sprague Dawley rats, originally acquired from the Center for Biomedical Research at the University of North Dakota. The subjects were approximately 12 months old at the start of the experiment. Each had experience leverpressing for sucrose and food pellet reinforcers delivered on interval schedules of reinforcement. They were maintained at approximately $85 \%$ of their free-feeding body weights by postsession feedings or by daily feeding on days on which sessions were not conducted. All the subjects were fed at the same time (after all the sessions had been completed). The subjects' free-feeding weights had been established prior to the experiment and were continuously maintained throughout the experiment. They were individually housed, with water freely available (only) in the home cage, and experienced a 12:12-h light:dark cycle with lights on at $0700 \mathrm{~h}$. The sessions were conducted during the light cycle.

Apparatus. Two identical Coulbourn Instruments experimental chambers for rats were used, each measuring $30.5 \times 25 \times 28.5 \mathrm{~cm}$ $(1 \times w \times h)$. Two 3.5-cm-wide $\times 0.1$-cm-thick response levers were located on the front panel. Each lever extended $2 \mathrm{~cm}$ into the chamber and could be depressed by a force of approximately $0.25 \mathrm{~N}$. The right lever was located $2.5 \mathrm{~cm}$ from the right wall; the left lever was $2.5 \mathrm{~cm}$ from the left wall. Both levers were $6.5 \mathrm{~cm}$ above the grid floor. A panel of three LED stimulus lights (red, yellow, and green) was located $5 \mathrm{~cm}$ above each lever. The lights were $0.6 \mathrm{~cm}$ in diameter, with the yellow light centered above the lever and the red and green lights $0.6 \mathrm{~cm}$ to the left and right, respectively. Centered on the front panel, $2 \mathrm{~cm}$ above the floor, was a $3.25-\mathrm{cm}$-wide $\times 3.75-\mathrm{cm}$ high $\times 2.5-\mathrm{cm}$-deep opening that allowed access to a trough into which reinforcers were delivered. Food pellets were delivered to the trough via a pellet dispenser located behind the front panel. Liquid sucrose was delivered to the trough via a syringe pump located outside the apparatus. A $1.5-\mathrm{cm}$-diameter houselight was centered on the back wall of the chamber, $2.5 \mathrm{~cm}$ below the ceiling.

Each chamber was housed in a sound-attenuating cubicle, with a ventilation fan masking noise from outside. Experimental events were programmed and data were recorded by an IBM-compatible personal computer running Graphic State software (Coulbourn Instruments) and connected to Coulbourn Instruments Habitest Universal Lincs. The control equipment was located in a room adjacent to the one housing the experimental chambers.

Procedure. Because the subjects were experimentally experienced, they were immediately started on the procedure. They were divided into two groups of 6 . The different groups experienced the identical procedure, with the lone exception that one responded for $1 \%$ liquid sucrose reinforcement, whereas the other responded for $5 \%$ sucrose.

The subjects responded in four conditions. In each, responding on the left lever during the first $25 \mathrm{~min}$ of the session was reinforced with $0.2 \mathrm{ml}$ of liquid sucrose (either $1 \%$ or $5 \%$ table sugar v/v with tap water) delivered by a RI 60 -sec schedule. Reinforcers were programmed at a probability of .01 every $0.6 \mathrm{sec}$, unless a reinforcer had already been programmed but not yet collected. In such an instance, the interreinforcer interval did not advance until the programmed reinforcer had been collected. Neither the session timer nor the interreinforcer interval advanced during reinforcer delivery. The red/left light above the left lever was illuminated, except during reinforcer delivery. The houselight was continuously illuminated.

The four conditions differed in terms of what occurred during the second $25 \mathrm{~min}$ of the session. In one condition $(1 \%-1 \% ; 5 \%-5 \%)$, the identical conditions of reinforcement were in effect during the second $25 \mathrm{~min}$. The reinforcement schedules in the different halves of the session were independent. Thus, if a reinforcer from the first half of the session had been programmed but not collected, it was canceled at the beginning of the second half. In the second condition $(1 \%-\mathrm{EXT} ; 5 \%-\mathrm{EXT})$, extinction was in effect during the second $25 \mathrm{~min}$. During this time, presses to the left lever were recorded but were not reinforced. No exteroceptive stimuli accompanied the transition between sucrose reinforcement and extinction (i.e., the red/left light above the left lever was illuminated during the final $25 \mathrm{~min}$ ). In the third condition ( $1 \%-\mathrm{FP} ; 5 \%-\mathrm{FP})$, the subjects responded for 45-mg food pellet reinforcers (P. J. Noyes, Formula A/I) in the second $25 \mathrm{~min}$. Again, no exteroceptive stimulus signaled the switch in reinforcer type, and any programmed reinforcers from the first half of the session were canceled. Food pellet reinforcers were delivered by an RI 60 -sec schedule. In the final condition $(1 \%-; 5 \%-\quad)$, the session terminated after $25 \mathrm{~min}$. 

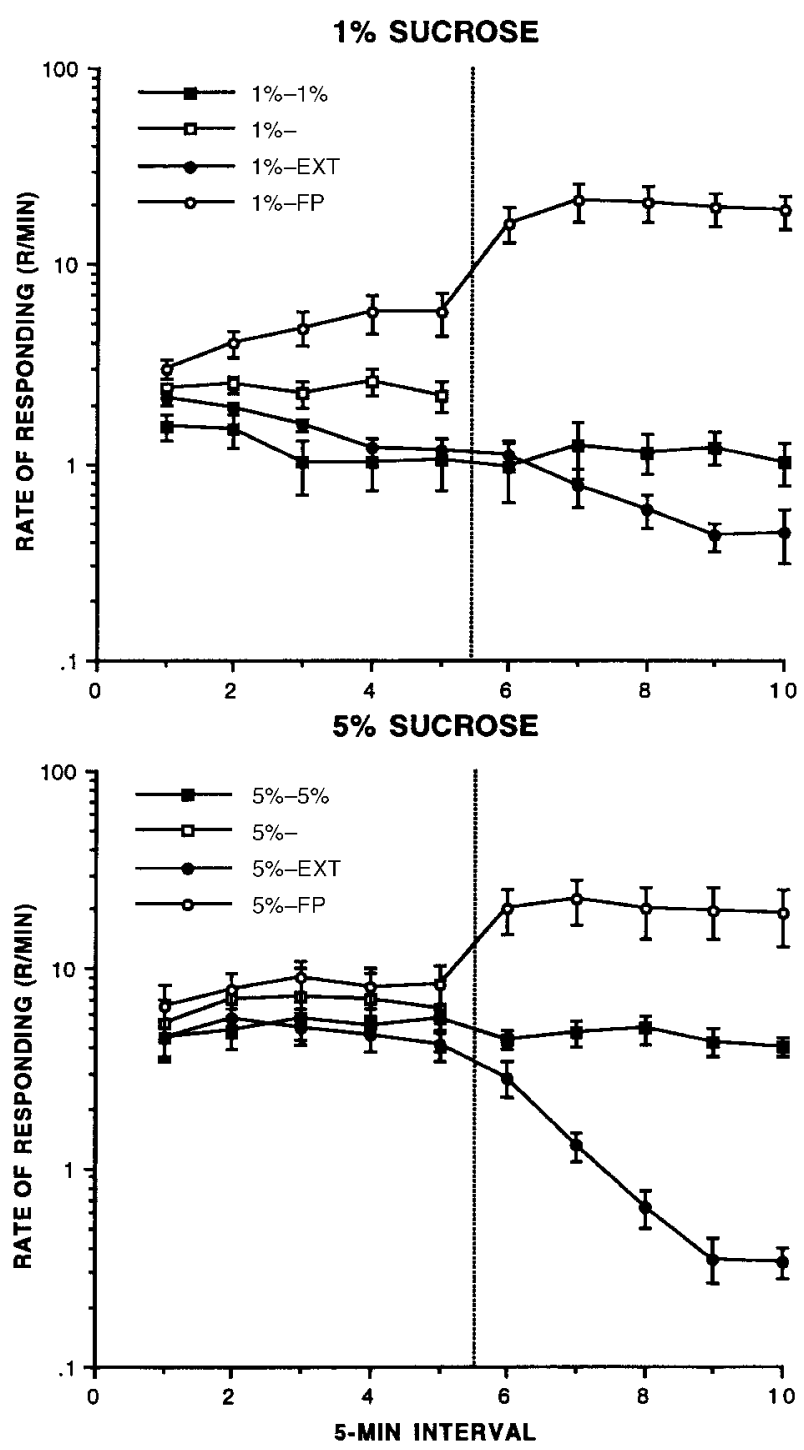

Figure 1. Mean rates of responding for all the subjects as a function of successive 5-min interval in the session for the subjects in the $1 \%$ (top graph) and $5 \%$ (bottom graph) sucrose groups in each of the four conditions in Experiment 1. Note the logarithmic ordinate.

Each condition was conducted for 20 consecutive sessions, with sessions conducted once per day, 5-6 days per week. In each group, 3 subjects experienced the conditions in the order listed above. The remaining 3 subjects experienced them in reverse order.

\section{Results and Discussion}

Figure 1 presents the rate of leverpressing across successive 5-min intervals of the 50-min session for the subjects in the $1 \%$ sucrose (top graph) and $5 \%$ sucrose (bottom graph) groups. Response rates are plotted on a logarithmic ordinate in this and all following figures so that differences in responding at low rates of responding are visually apparent. Each function represents the mean rate of responding for all the subjects, calculated using the final five sessions of the condition. The error bars represent the standard error of the mean across subjects responding in that particular 5-min interval. In this and all following figures that present responding from 50-min sessions, the dashed line in each graph symbolically separates responding in the first and the second halves of the session.

The results in Figure 1 indicate that the conditions of reinforcement in the second half of the session controlled responding in both halves. The lowest and highest rates of responding in the second half of the session were observed when extinction and food pellet reinforcement, respectively, were in effect. The highest rates of responding in the first half of the session were observed when food pellets would be available in the second half.

The data in Figure 1 were analyzed by conducting a three-way (group $\times$ condition $\times 5$-min interval) mixed model analysis of variance (ANOVA) on the response rates (not logarithms) of individual subjects. The results from this and all following analyses were considered significant at $p<.05$. The main effect of group was significant $[F(1,10)=7.06]$, indicating that $5 \%$ sucrose maintained higher rates of responding than did $1 \%$ sucrose. The main effect of condition was significant $[F(3,30)=$ 8.20 ], indicating that responding changed with changes in the upcoming conditions of reinforcement. The main effect of 5-min interval was also significant $[F(4,40)=$ 2.71], indicating that response rates changed across the first half of the session. Finally, the interaction between condition and 5 -min interval was significant $[F(12,120)=$ 4.32]. No other effects reached, or approached, significance.

Because no interactions involving the group variable were significant, follow-up analyses collapsed across groups. Three two-way (condition $\times 5$-min interval) repeated measures ANOVAs were conducted to determine whether having no second half, extinction in the second half, or food pellet reinforcement in the second half of the session altered responding in the first half, relative to when sucrose would be available in the second half. When the sucrose-only condition was compared with the nosecond-half condition, the main effect of condition, the main effect of 5-min interval, and the interaction term failed to reach significance. When the sucrose-only condition was compared with the sucrose-extinction condition, only the interaction term $[F(4,44)=3.81]$ reached significance. Tests for simple effects indicated that response rates in (only) the fifth 5-min interval were significantly lower in the sucrose-extinction condition than in the sucrose-only condition $[F(1,11)=5.34]$. Together, these results suggest that sucrose reinforcement in the second half of the session did not suppress responding for sucrose in the first half.

The final analysis compared the sucrose-only condition with the sucrose-food-pellet condition. The main effect of condition $[F(1,11)=18.44]$, the main effect of 5-min interval $[F(4,44)=4.52]$, and the interaction term $[F(4,44)=$ 3.75] were significant. Tests for simple effects indicated that responses rates in the sucrose-food-pellet condition were higher than those in the sucrose-only condition during each 5-min interval in the first half of the session [all 
$\left.F_{\mathrm{s}}(1,11) \geq 7.12\right]$, thus indicating that upcoming food pellet reinforcement increased response rates for sucrose in the first half of the session.

The results of Experiment 1 provide little evidence to support the idea that sucrose reinforcement in the second half of a session suppresses response rates for sucrose in the first half. In fact, they provide fairly strong evidence that food pellet availability in the second half of a session increases sucrose-reinforced response rates in the first half. However, as with previous studies, the results do not specifically pinpoint upcoming food pellets as the cause of this induction.

\section{EXPERIMENT 2}

Experiment 2 was designed to determine whether induction is controlled by prospective or retrospective factors. That is, the subjects' response rates for sucrose may increase because food pellet reinforcement is upcoming. Alternatively, they may increase because food pellets were delivered in the previous session.

To delineate between these possibilities, Experiment 2 was modeled after an experiment by Flaherty and Rowan (1985). They investigated negative consummatory contrast, which occurs when the consumption of one substance low in hedonic value is suppressed when it will be followed by an opportunity to consume a substance with a high hedonic value, relative to when it is followed by itself. To determine whether the suppressed consumption was prospective or retrospective, they conducted a withinsubjects experiment that employed two types of session, each associated with distinctly different discriminative stimuli, presented in strict alternation. In one, the subjects had two successive opportunities to consume $0.15 \%$ saccharin. In the other, the opportunity to consume $0.15 \%$ saccharin was followed by the opportunity to consume $32 \%$ sucrose (which is preferred to $0.15 \%$ saccharin). Consumption of saccharin during the initial period was lower during sessions in which $32 \%$ sucrose, rather than saccharin, was upcoming. Because session types alternated, their results supported the idea that the phenomenon was anticipatory (i.e., prospective).

Experiment 2 also employed a within-subjects design with two types of session. In one, leverpressing was reinforced with liquid sucrose in both halves of the session. In the other, liquid sucrose reinforcers were delivered in the first half of the session, and food pellet reinforcers were delivered in the second. These sessions were presented in strict alternation and were associated with distinct discriminative stimuli (i.e., the location of the lever used during the session and the color/location of the stimulus lights).

The results of Experiment 2 could potentially delineate between the retrospective and the prospective influences of food pellets in two ways. The first was documenting which type of session produced the highest rates of responding in the first half of the session. If induction is retrospective and occurs because food pellets were available in the previous session, then the highest rates of responding in the first half of the session should occur in sessions in which food pellet reinforcement is not upcoming, because these sessions are always preceded by a session in which food pellets were delivered. However, if induction is prospective and occurs because food pellets will be available in the current session, then response rates in the first half of the session should be highest in sessions in which food pellets will be available in the second half.

The second form of delineation is documenting how quickly differential responding develops in the different types of session. If induction occurs because food pellets were delivered in the previous session, the influence of food pellets should be fairly immediate, because its appearance would not require the subjects to learn about the types of session. However, if induction occurs because food pellets will be available in the current session, it should take time to develop, because the effect will rely on the subjects' learning that, in certain sessions, food pellet reinforcement will be available in the second half of the session (see Timberlake \& Engle, 1995, for similar reasoning).

\section{Method}

Subjects and Apparatus. The subjects were 12 experimentally naive male Sprague Dawley rats obtained, housed, and, with one exception, maintained as were those in Experiment 1. The subjects were approximately 4 months old at the start of the experiment, and their free-feeding body weights had yet to be established. To do so, they were given free access to food for 2 weeks. Free-feeding weights were then determined by a single weighing. The subjects' $85 \%$ weights were then calculated and, once reached, were continuously maintained.

The subjects responded in an apparatus that was identical to those used in Experiment 1, with the exception that the two response levers could be retracted from the chamber.

Procedure. Leverpress training was accomplished by placing the subjects in the apparatus with only one (right or left) lever available and reinforcing leverpressing on a continuous reinforcement schedule. The reinforcer was a 45-mg food pellet. Once each subject had pressed each lever more than 100 times, the experiment began.

The subjects responded in 50-min sessions. During the first $25 \mathrm{~min}$, leverpressing was reinforced with $0.2 \mathrm{ml}$ of liquid sucrose delivered by an RI 60-sec schedule, with reinforcers programmed as in Experiment 1. During the second $25 \mathrm{~min}$, the reinforcer was either the same sucrose reinforcer as that delivered in the first $25 \mathrm{~min}$ or a 45-mg food pellet. Reinforcers in the second half of the session were also delivered by an RI 60-sec schedule. Distinct discriminative stimuli accompanied each type of session. In one, the subjects pressed the left lever in the presence of the red/left stimulus lights. In the other, they pressed the right lever in the presence of the green/right stimulus lights. For each session type, the opposite lever was retracted from the chamber, but the appropriately colored/ positioned stimulus light was illuminated above the retracted lever. In all sessions, the stimulus lights were extinguished during reinforcer delivery. Session type alternated strictly, with the subjects responding in a total of 40 sessions (i.e., 20 of each type).

The subjects were divided into two groups of 6 . One group responded for $1 \%$ liquid sucrose reinforcers, whereas the second group responded for $5 \%$ sucrose. For 3 subjects in each group, the left lever and red/left stimulus lights were present in sessions in which sucrose reinforcement was available throughout the session, and the right lever and green/right stimulus lights were present in sessions in which sucrose and food pellet reinforcers were available 

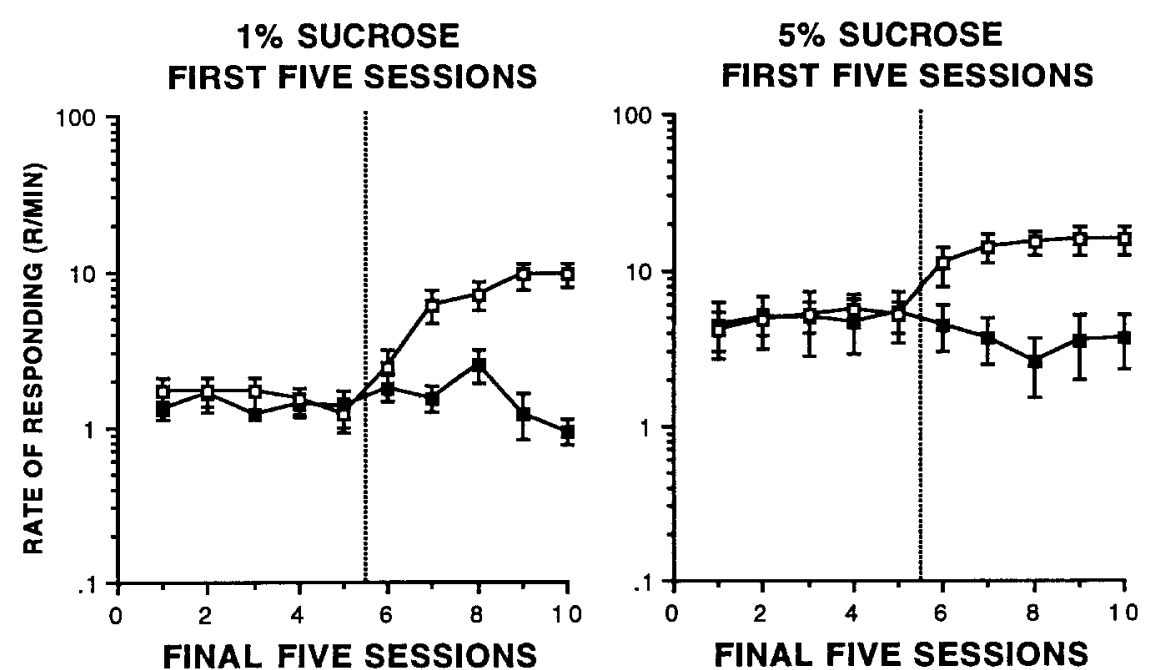

FINAL FIVE SESSIONS
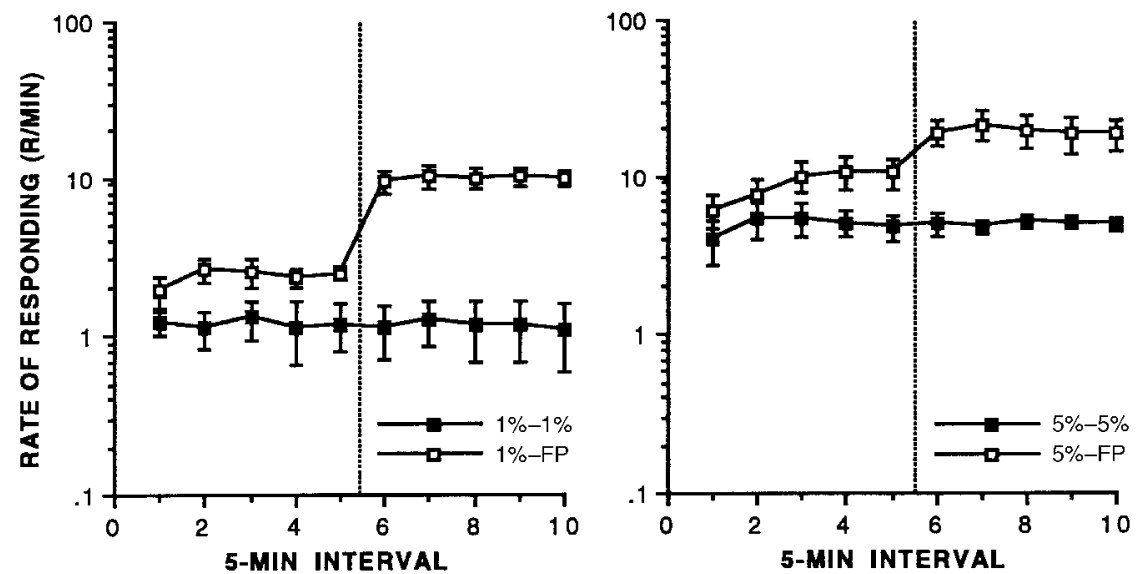

Figure 2. Mean rates of responding for all the subjects as a function of successive 5-min interval in the session for the subjects in the $1 \%$ (left column) and $5 \%$ (right column) sucrose groups in the sucrose-only ( $1 \%-1 \%$ or $5 \%-5 \%$; closed squares) or the sucrose-food-pellet (1\%-FP or 5\%-FP; open squares) sessions in Experiment 2. The top graphs present results from the initial five sessions of each type of session, whereas the bottom graphs present results from the final five sessions of training. Note the logarithmic ordinate.

in the first and second halves, respectively, of the session. The other 3 subjects in each group received the reverse conditions. Sessions were conducted daily, 5-6 days per week.

\section{Results and Discussion}

Figure 2 presents the mean rates of leverpressing across successive 5-min intervals of the session for all the subjects responding for $1 \%$ (left column) or $5 \%$ (right column) sucrose. Individual functions represent the results from sessions in which sucrose reinforcers were delivered throughout the session (closed squares) or in which sucrose and food pellet reinforcers were delivered in the first and the second, respectively, $25 \mathrm{~min}$ of the session (open squares). The error bars represent the standard error of the mean across subjects for that particular 5-min interval. The top row of graphs presents responding in the first five sessions of each type. The bottom row of graphs presents responding in the final five sessions of each type. The data in Figure 2 suggest that differential responding in the first half of the session was not present early in training but was present at the end of training. More important, response rates in the first half of the session were higher in sessions in which food pellet reinforcers would be available in the second half than in sessions in which sucrose reinforcers would be available.

Statistical analyses supported these conclusions. Responding in the first half of the session in the initial five sessions was analyzed by conducting a three-way (group $\times$ type of session $\times 5$-min interval) mixed model ANOVA. The main effect of group was significant $[F(1,10)=10.49]$, indicating that the subjects in the $5 \%$ sucrose group responded at a higher rate than did those in the $1 \%$ sucrose group. No other effects reached (or approached) significance, indicating that responding in the different sessions did not differ early in training. Responding in the first half of the session during the final five sessions was analyzed by conducting an identical analysis. The results indicated that the main effect of group was again significant $[F(1,10)=$ 
12.45]. In this analysis, however, the main effect of type of session $[F(1,10)=18.33]$, the main effect of 5-min interval $[F(4,40)=4.76]$, the interaction between type of session and 5-min interval $[F(4,40)=3.24]$, the interaction between type of session and group $[F(1,10)=5.66]$, the interaction between 5-min interval and group $[F(4,40)=3.51]$, and the three-way interaction $[F(4,40)=2.74]$ were also significant.

Because of the significant interactions involving the group variable, follow-up analyses were conducted separately for the two groups. Response rates for the $1 \%$ sucrose group in the first half of the session were analyzed by a two-way (type of session $\times 5$-min interval) repeated measures ANOVA. The results showed that the main effect of type of session was significant $[F(1,5)=67.34]$, indicating that higher rates of responding for $1 \%$ sucrose were observed in the first $25 \mathrm{~min}$ of the session when food pellet, rather than sucrose, reinforcers would be delivered in the second $25 \mathrm{~min}$. However, the main effect of 5-min interval and the interaction term were not significant. An identical analysis of response rates for the 5\% sucrose group showed that the main effect of session type $[F(1,5)=$ 11.09], the main effect of 5-min interval $[F(4,20)=4.05]$, and the interaction term $[F(4,20)=3.03]$ were significant. Tests for simple effects indicated that rates of responding were higher at each 5-min interval in the first half of the session in sessions in which food pellet, rather than $5 \%$ sucrose, reinforcement would be delivered in the second half [all $F$ s $(1,5) \geq 6.76]$.

Two pieces of evidence from Experiment 2 suggest that, in the present procedure, induction was controlled by prospective factors. First, the highest response rates for sucrose in the first half of the session were observed in sessions in which food pellet reinforcement would be available in the second half, despite the fact that this type of session was always preceded by one in which food pellets had not been delivered. Second, differential responding took time to develop. If induction occurred because food pellets had been delivered in the previous session, differential responding should have occurred very early in training. But it did not.

Although the results support a prospective explanation, they do not rule out retrospective ones. It is possible that the subjects learned to increase their response rates in the first half of the sucrose-food-pellet sessions because food pellets were not delivered in the second half of the previous session. In fact, the retrospective influence of the previous session may have emerged only after the subjects learned how the sessions alternated.

\section{EXPERIMENT 3}

In Experiment 2, induction occurred in sessions in which food pellet reinforcement would be delivered in the second half of the session, despite the fact that these sessions always followed ones in which food pellets were not available. These results seem to rule out the possibility that induction occurred because food pellets were delivered and consumed in the previous session, but they do not rule out the idea that the previous session contributed to the induction. The subjects may have learned that sessions in which food pellet reinforcement would be available always followed sucrose-only sessions. In other words, the induction may indeed have been controlled by retrospective factors.

In the initial phase of Experiment 3, this possibility was assessed. It replicated the procedure of Experiment 2, with the exception that, instead of strictly alternating sessions, session type varied pseudorandomly. With this design, the previous session ceases to be an accurate predictor of the conditions of reinforcement in the present session. If retrospective influences played a role in the induction observed in Experiment 2, induction should not be observed in Experiment 3, because the procedure should remove those influences. If prospective influences control the induction, however, a similar induction effect should be observed in Experiment 3, because each session type was correlated with distinct discriminative stimuli.

Even if prospective processes control the induction, one still needs to concede that the causal factors for behavior cannot occur subsequent to the behavior itself. Rather, something present in the subjects' environment must cause the induction (e.g., a stimulus that produces an "expectancy" of upcoming food pellet reinforcement). Several such potential environmental variables exist. One may be the conditions of reinforcement in the previous session. As was noted above, the subjects may have used the previous session to predict the conditions in the current one. Other potential variables include the lever location and/or the stimulus lights correlated with the different types of session. It is also possible that these and/or other variables combined to produce the induction (e.g., sucrose reinforcers and/or the passage of time in the presence of the green/right light $=$ upcoming food pellets-i.e., an establishing operation; Michael, 1993).

Besides trying to determine the influence of the previous session, Experiment 3 also determined which, if any, of these stimuli might control the appearance of induction. As was noted above, the subjects initially responded in training sessions identical to those used in Experiment 2 that varied pseudorandomly. After experiencing 40 such sessions, the subjects responded in a series of 25-min probe sessions in which both levers were available and, across probe sessions, the placement of the stimulus lights and the presence of sucrose reinforcement were varied. If induction is prospective and the lever location and/or the stimulus lights correlated with the different types of sessions control it, then responding in the probe sessions should document this control. Specifically, if lever location signals upcoming food pellet reinforcement, then rates of responding in the probe sessions should be higher on the lever used in sessions with food pellet reinforcement than on the lever used in the sucrose-only sessions. Likewise, if the stimulus lights are used to signal upcoming food pellet reinforcement, then responding in the probe sessions should be controlled by the stimulus lights, not by lever location.

In addition, because the length of the probe sessions was equal to that of the first half of the training sessions, it was 


\section{FINAL FIVE SESSIONS $1 \%$ SUCROSE}
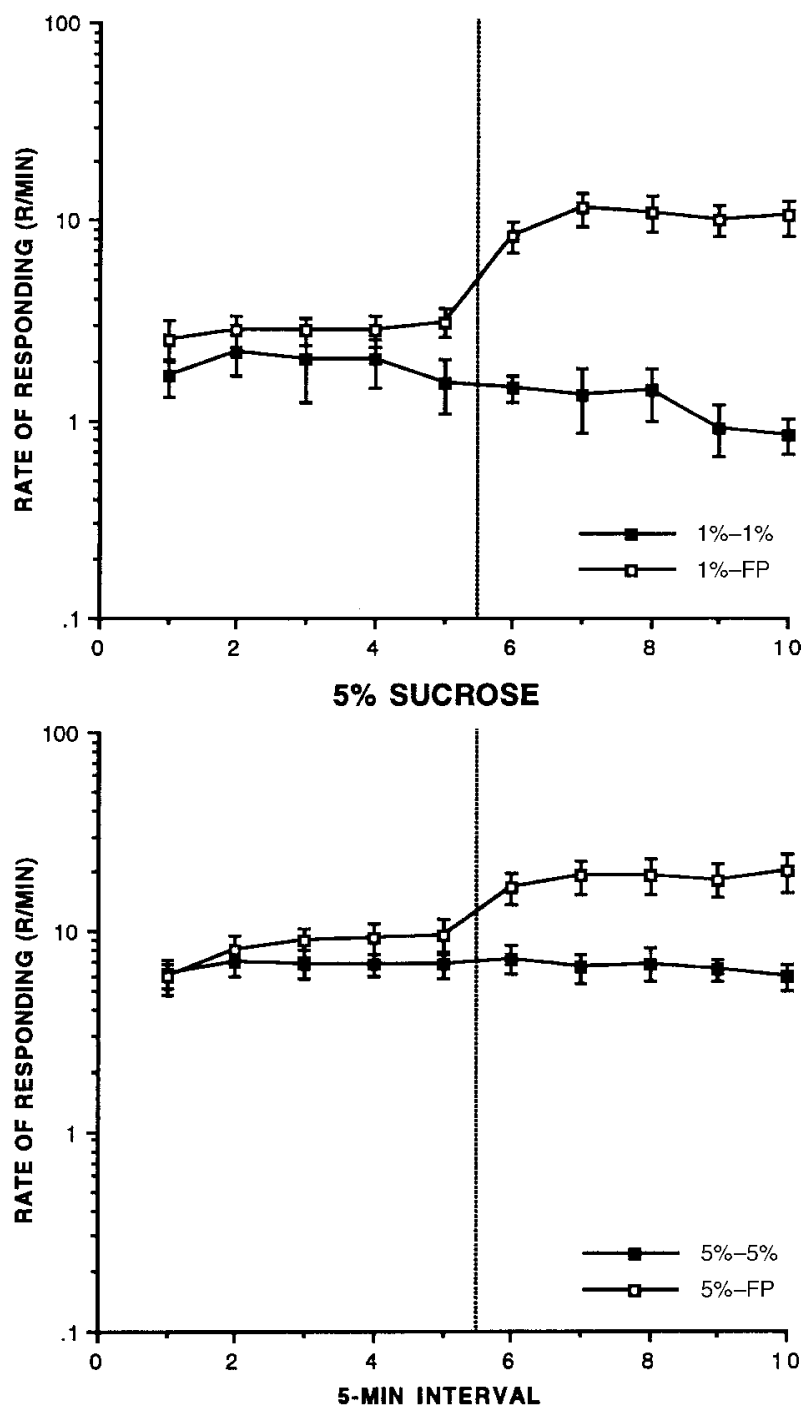

Figure 3. Mean rates of responding for all the subjects as a function of successive 5-min interval in the sessions for the subjects in the $1 \%$ (top graph) and $5 \%$ (bottom graph) sucrose groups in the sucrose-only (1\%-1\% and $5 \%-5 \%$; closed squares) and sucrose-food-pellet (1\%-FP or 5\%-FP; open squares) sessions of Experiment 3. Note the logarithmic ordinate.

possible to identify temporal control over responding (i.e., changes in responding as a function of time). For instance, the subjects may have attended to the above-mentioned stimuli only as the midpoint of the (training) session approached. If so, one would predict that differences would be found in responding late, but not early, in the probe sessions.

\section{Method}

Subjects and Apparatus. The subjects were 12 experimentally experienced male Sprague Dawley rats. They were obtained, housed, and maintained as were those in Experiment 1. They were not those used in Experiment 1 (or 2) but did have similar experimental expe- rience. The subjects responded in the same apparatus as that used in Experiment 2.

Procedure. Because the subjects were experimentally experienced, they were started directly on the procedure. For the first 40 sessions, the subjects responded in the two types of session employed in Experiment 2. The only difference from Experiment 2 was that, instead of the sessions alternating strictly, session order across the first 40 sessions was determined pseudorandomly (independently for each subject). The following constraints were in effect when determining session order. First, no session type could occur for more than 3 consecutive sessions. Second, 20 sessions of each type of session had to occur in the 40 sessions.

As in Experiment 2, the subjects were divided into two groups of 6 , with one group responding for $1 \%$ sucrose and the other for $5 \%$ sucrose. Also as in Experiment 2, for 3 subjects in each group, the left lever and the red/left stimulus lights were associated with one type of session, and the right lever and the green/right stimulus lights were associated with the other type. The remaining subjects received the opposite pairings.

Starting with Session 41, the subjects responded in a series of 25min probe sessions in which both levers were available throughout the session. Sessions of 25 min were chosen because they equaled the length of the first half of the training sessions. Four types of probe sessions were conducted. In two, a concurrent RI 60-sec RI 60 -sec schedule was in effect, with both levers delivering the same sucrose reinforcer as that received during training (i.e., $1 \%$ or $5 \%$ sucrose). A 2-sec changeover delay was in effect for switching the levers, during which responses were recorded but not reinforced. The two probe sessions differed in the placement of the stimulus lights. In one, the red/left stimulus light was illuminated above the left lever, and the green/right light was illuminated above the right lever. In the other, this placement was reversed. In other words, in one probe session, the stimulus lights appeared over the lever that had been inserted in their presence during the training sessions. In the other, they appear over the opposite lever. (Note that, in training sessions, stimulus lights with the same color/location were illuminated over both the inserted and the retracted lever.)

In the other two probe sessions, extinction was in effect. These sessions also differed in whether the colored stimulus light (red/left or green/right) was illuminated over the same or the opposite lever as in training.

The subjects responded twice in each type of probe session. Thus, eight total probe sessions were conducted, the order of which was determined randomly, independently for each subject. Probe sessions were separated by four training sessions (i.e., 50-min sessions), with each of the two types of original training sessions occurring twice across those four sessions (the order of which was determined randomly, with the constraint that each type of session had to occur twice).

\section{Results and Discussion}

Figure 3 shows that induction was observed despite the fact that session type alternated pseudorandomly. It presents the rate of leverpressing as a function of successive 5-min interval in the 50-min session over the final five sessions in which sucrose reinforcers were delivered in both halves of the session or sucrose and food pellet reinforcers were delivered in the first and the second halves, respectively, of the session. The top graph presents the results for the $1 \%$ sucrose group. The bottom graph presents the results for the $5 \%$ sucrose group. The error bars again represent the standard error of the mean across subjects.

The results from statistical analyses supported the conclusion that induction was observed. Response rates from the first half of the session were analyzed by a three-way 
(group $\times$ type of session $\times 5$-min interval) mixed model ANOVA. The main effect of group was significant $[F(1,10)=16.89]$, indicating that $5 \%$ sucrose maintained higher rates of responding than did $1 \%$ sucrose. The main effect of type of session was significant $[F(1,10)=14.75]$, indicating that response rates were higher when food pellet, rather than sucrose, reinforcement was upcoming. The main effect of 5-min interval $[F(4,40)=3.79]$ and the interaction between type of session and 5-min interval $[F(4,40)=4.24]$ were both significant, indicating that responding changed across the first half of the session and did so differently in the different sessions. No other effects were significant. Follow-up analyses on the significant interaction indicated that response rates were higher when food pellets were upcoming than when sucrose was upcoming in all but the first 5-min interval in the first half of the session [all $F_{\mathrm{S}}(1,11) \geq 7.38$ ].

The results shown in Figure 3 suggest that the induction reported in Experiment 2 was not the product of the strict alternation of session type. In Experiment 3, ses- sions that provided food pellet reinforcement were equally likely to be preceded by either type of session. However, induction was still observed. The present results do not, however, altogether rule out the possibility that retrospective factors can, in some instances, contribute to induction. In fact, a visual comparison of the bottom graphs in Figure 2 and the graphs in Figure 3 provides some indication that they can. The size of induction in Experiment 3 appears to have been smaller than that in Experiment 2. A close inspection suggests that this reduction in size may have been due to higher rates of responding in the sucroseonly sessions in Experiment 3 than in Experiment 2. This result may indicate that, in the absence of information from the previous session, response rates were increased in the first half of both types of session. It should also be noted that in the present procedure, as in Flaherty and Rowan (1985), distinct stimuli were correlated with each type of session. Retrospective influences have been shown to be more influential in the absence, than in the presence, of such stimuli (Timberlake \& Engle, 1995).

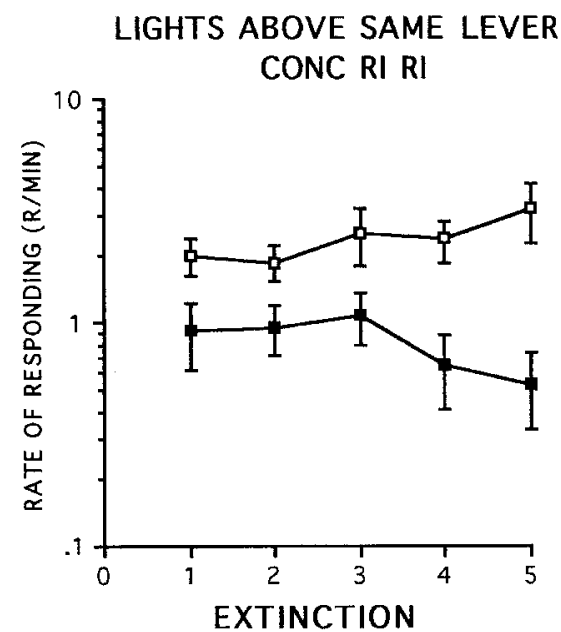

\section{LIGHTS ABOVE OPPOSITE LEVER CONC RI RI}

10
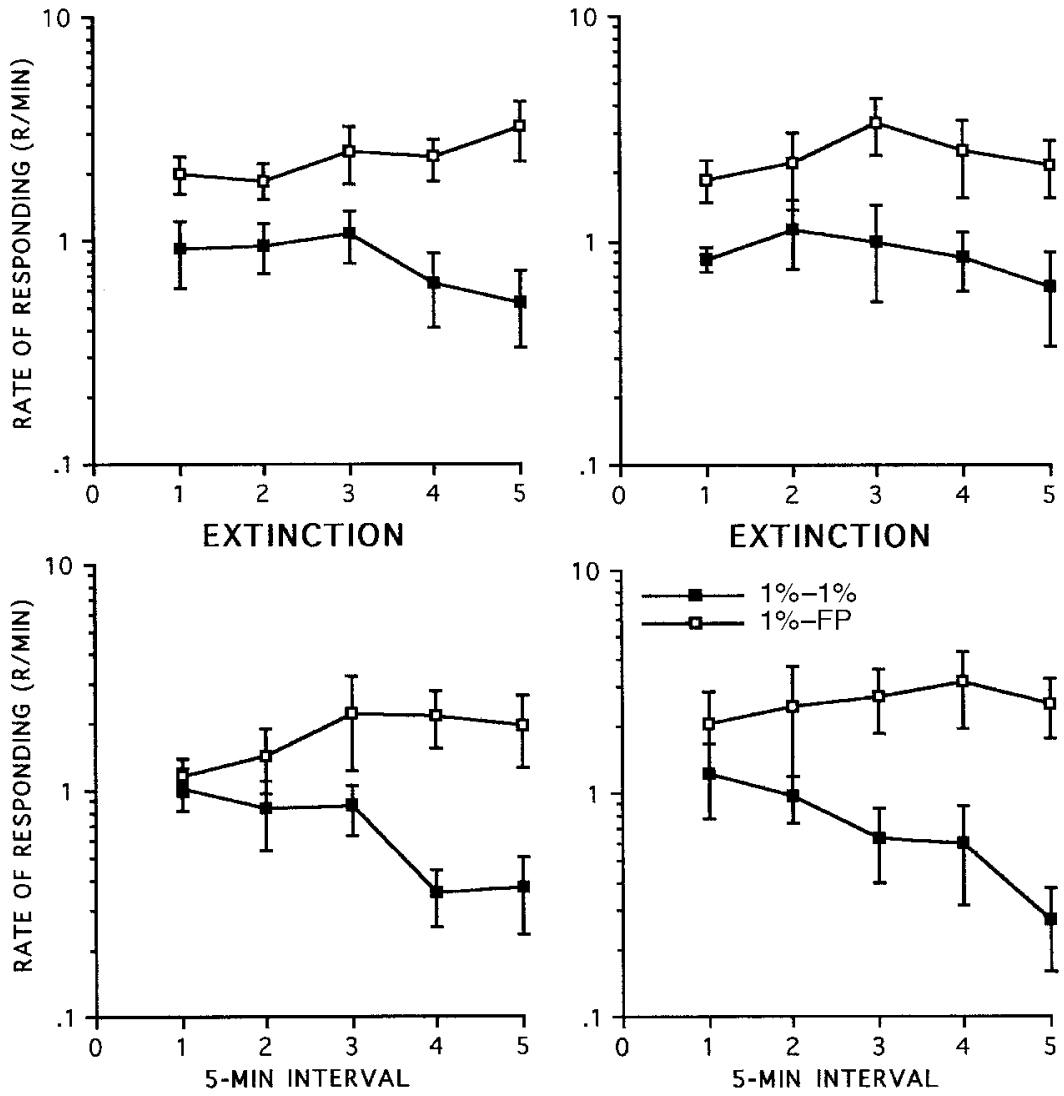

Figure 4. Mean rates of responding in the probe sessions on the lever used in the sucroseonly (1\%-1\%; closed squares) and sucrose-food-pellet (1\%-FP; open squares) sessions for all the subjects in the $1 \%$ sucrose group during sessions in which a concurrent (CONC) random interval RI RI schedule (top graphs) or extinction (bottom graphs) was in effect and the colored stimulus lights appeared over the same (left column) or the opposite (right column) lever as that during training. Note the logarithmic ordinate. 
Figure 4 presents the results from the four types of probe sessions for the subjects in the $1 \%$ sucrose group. Rates of leverpressing are presented as a function of successive 5-min interval in the 25-min session. Figure 4 was constructed similarly to Figure 3, with the exception that the data were averaged across the two presentations of each probe session.

The data in Figure 4 indicate that, across the four types of probe sessions, the subjects in the $1 \%$ sucrose group responded at a higher rate on the lever used in training sessions in which food pellet reinforcement was upcoming than on the lever used in the sucrose-only sessions. The results from a four-way (reinforcement schedule $\times$ stimulus light $\times$ lever $\times 5$-min interval) repeated measures ANOVA showed a significant main effect of lever $[F(1,5)=$ 14.07], indicating that the subjects responded at a higher rate on the lever used in the sucrose-food-pellet sessions than on the one used in the sucrose-only sessions. It also produced a significant interaction between lever and 5min interval $[F(4,20)=5.05]$, indicating that rates of re- sponding changed differently across the 25 -min session on the different levers, suggesting some measure of temporal control over responding. All other effects failed to reach significance.

Figure 5 presents the results from the probe sessions for the subjects in the 5\% sucrose group. It was constructed as was Figure 4, and although the overall size of the induction effect was not as large, it indicated that lever location again controlled response rates. Results from a four-way (reinforcement schedule $X$ stimulus light $X$ lever $\times 5$-min interval) repeated measures ANOVA showed that the main effect of reinforcement schedule $[F(1,5)=$ 31.19] was significant, indicating that the subjects responded at a higher rate during the concurrent RI RI sessions than during the extinction sessions. The main effect of lever was also significant $[F(1,5)=11.16]$, indicating that the subjects responded at a higher rate on the lever used in the sucrose-food-pellet sessions than on the one used in the sucrose-only sessions. The interaction between reinforcement schedule and 5-min interval was also sig-
LIGHTS ABOVE SAME LEVER CONC RI RI
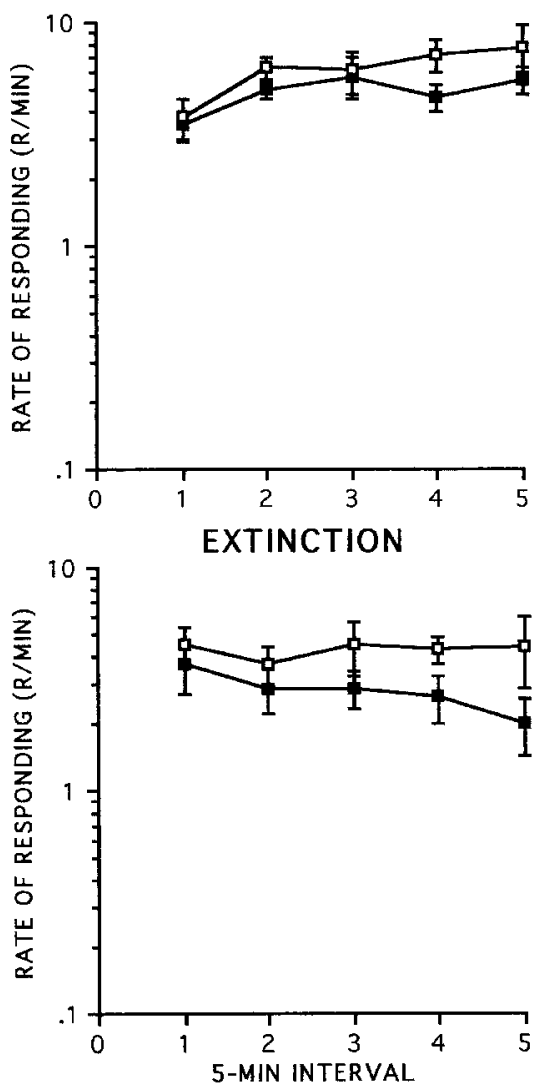

LIGHTS ABOVE OPPOSITE LEVER CONC RI RI
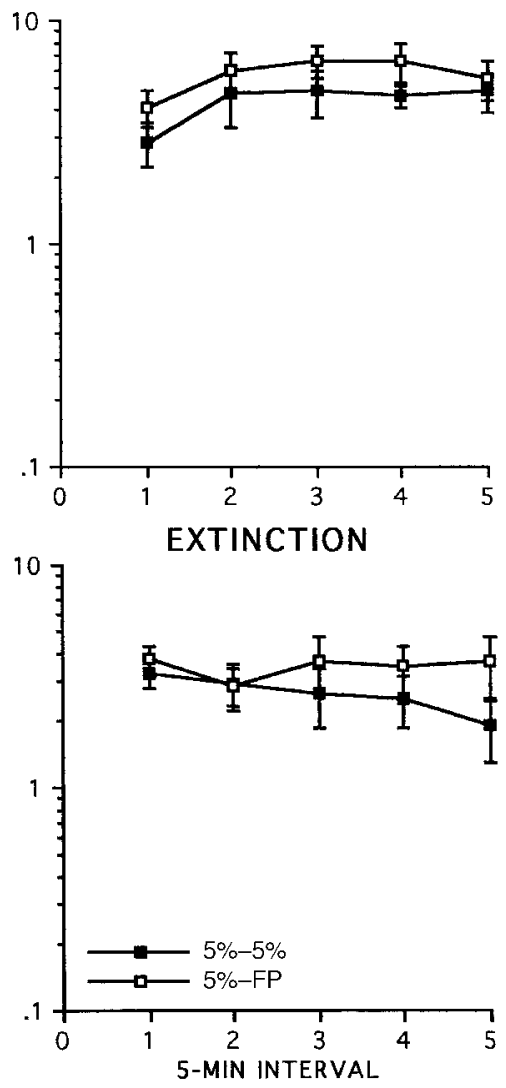

Figure 5. Mean rates of responding in the probe sessions on the lever used in the sucroseonly (5\%-5\%; closed squares) and sucrose-food-pellet (5\%-FP; open squares) sessions for all the subjects in the $5 \%$ sucrose group during sessions in which a concurrent (CONC) random interval RI RI schedule (top graphs) or extinction (bottom graphs) was in effect and the colored stimulus lights appeared over the same (left column) or the opposite (right column) lever as that during training. Note the logarithmic ordinate. 
nificant $[F(4,20)=18.93]$, indicating that response rates changed differently across the 25 -min session as a function of reinforcement schedule. All other effects failed to reach significance.

Figures 4 and 5 and the corresponding statistical analyses indicate that the induction shown in Figure 3 was controlled by lever location. The subjects responded at a higher rate on the lever that was, in training sessions, used to obtain food pellet reinforcement than on the lever used in sucroseonly sessions. These results do not, however, identify the reason why lever location controlled responding.

\section{EXPERIMENT 4}

The results of Experiment 3 suggest that the subjects discriminated the type of session and, thus, the upcoming reinforcer on the basis of lever location. Such a finding should not be unexpected. For instance, these results are consistent with those of Flaherty and Rowan (1985), who found negative consummatory contrast between sessions associated with different discriminative stimuli. Unlike their results, however, Experiment 3 showed that this discrimination led to an increase, not a decrease, in responding.

One potential explanation for increased responding on the lever used to obtain the upcoming food pellets is that, through Pavlovian conditioning, the lever became a conditioned exciter. Because lever location was reliably paired with food pellet reinforcement, its presence may have elicited a conditioned response. That response may have come in the form of sign tracking (Hearst \& Jenkins, 1974), in that the subjects approached and contacted the lever, or it may have been more general (e.g., conditioned arousal; Killeen, 1995).

Although the results of Experiment 3 are consistent with this idea, it is not the only potential explanation. For instance, the subjects may have favored one lever over the other simply because pressing one (i.e., the lever used in sucrose-food-pellet sessions) produced a higher overall level of reinforcement than did pressing the other.

Experiment 4 tested whether the results in Experiment 3 were the outcome of Pavlovian processes by having the subjects respond in sessions in which the location of the lever, either right or left, used in the first half of the session was predictive of the type of reinforcer that would be available in the second half. Unlike in the previous procedure, however, the reinforcer available in the second half of the session, either sucrose or a food pellet, was equally likely to be obtained by pressing either the right or the left lever. Thus, lever location in the first half of the session was perfectly correlated with a certain upcoming reinforcer, but each lever was equally correlated with both sucrose and food pellet reinforcement. If the induction observed in Experiment 3 was the outcome of Pavlovian processes, then the present procedure should lead to results similar to those observed in Experiment 3. On the other hand, if other factors, such as lever location being differentially correlated with overall reinforcement, control the induction, then it should be absent.

\section{Method}

Subjects and Apparatus. The subjects were 12 experimentally experienced male Sprague Dawley rats obtained, housed, and maintained as were those in Experiment 1. They also had similar previous experience. They were not those used in Experiments 1-3. One group (see below) responded in the apparatus used in Experiment 2. The second group responded in an identical apparatus.

Procedure. Because the subjects were experimentally experienced, they were started directly on the procedure. As in the previous experiments, the subjects were divided into two groups of 6 . One group responded for $1 \%$ sucrose reinforcement, and the other responded for $5 \%$ sucrose. For the first 80 sessions, the subjects responded in sessions similar to those in Experiment 2. In one (SUCSUC), sucrose reinforcers were delivered by an RI 60-sec schedule during both halves of the 50-min session. In the other (SUC-FP), sucrose reinforcers were delivered in the first half of the session, and a food pellet served as the reinforcer in the second half. For each type of session, the reinforcer in the first half of the session was delivered for responding on a particular lever. Three subjects in each group responded on the left lever in the first half of the SUC-SUC sessions. The other 3 responded on the right lever in the first half of the SUC-SUC sessions. At the midpoint of the session, the lever was retracted, and $0.2 \mathrm{sec}$ later, either the right or the left lever was inserted into the chamber. Depending on the type of session, sucrose or food pellet reinforcement was earned for the remainder of the session by pressing the newly inserted lever. During all the sessions, the red/left light above the active lever was illuminated throughout the session, except during reinforcer delivery.

Thus, the subjects experienced four particular types of session (with the active lever during the second half of the session in parentheses): SUC-SUC (right), SUC-SUC (left), SUC-FP (right), and SUC-FP (left). The subjects experienced these sessions in pseudorandom order, determined independently for each subject, using the following constraints: No particular one of the four session types could be presented more than two times in succession (although a particular type of reinforcer in the second half could be presented in more than two successive sessions), and each session type had to occur exactly 20 times in the 80 training sessions.

Beginning with Session 81, the subjects responded in two types of 25-min probe sessions in which both levers were available. In one, responding was reinforced on a concurrent RI 60-sec RI 60-sec schedule, with sucrose reinforcers programmed identically to the concurrent schedule probe sessions in Experiment 3. In the other, extinction was in effect. In both types of probe sessions, the red/left light above each lever was illuminated, except during reinforcer delivery. Each subject responded twice in each type of probe session. Order of probe session presentation was determined randomly, independently for each subject. Probe sessions were separated by four training (i.e., 50-min) sessions, one of each type described above, the order of which was randomly determined.

\section{Results and Discussion}

Figure 6 shows that induction was not observed in the training sessions for either group. It was constructed similarly to Figure 3, using the final five SUC-SUC and SUC-FP sessions (regardless of which lever was in use in the second half of the session). Responding in the first half of the session was analyzed by a three-way (group $\times$ type of session $\times 5$-min interval) mixed model ANOVA. The main effect of group was significant $[F(1,10)=$ $11.00]$, indicating that $5 \%$ sucrose maintained higher response rates than $1 \%$ sucrose. The main effect of 5-min interval was also significant $[F(4,40)=3.30]$, indicating that response rates changed across the first half of the session. No other effects were significant. 

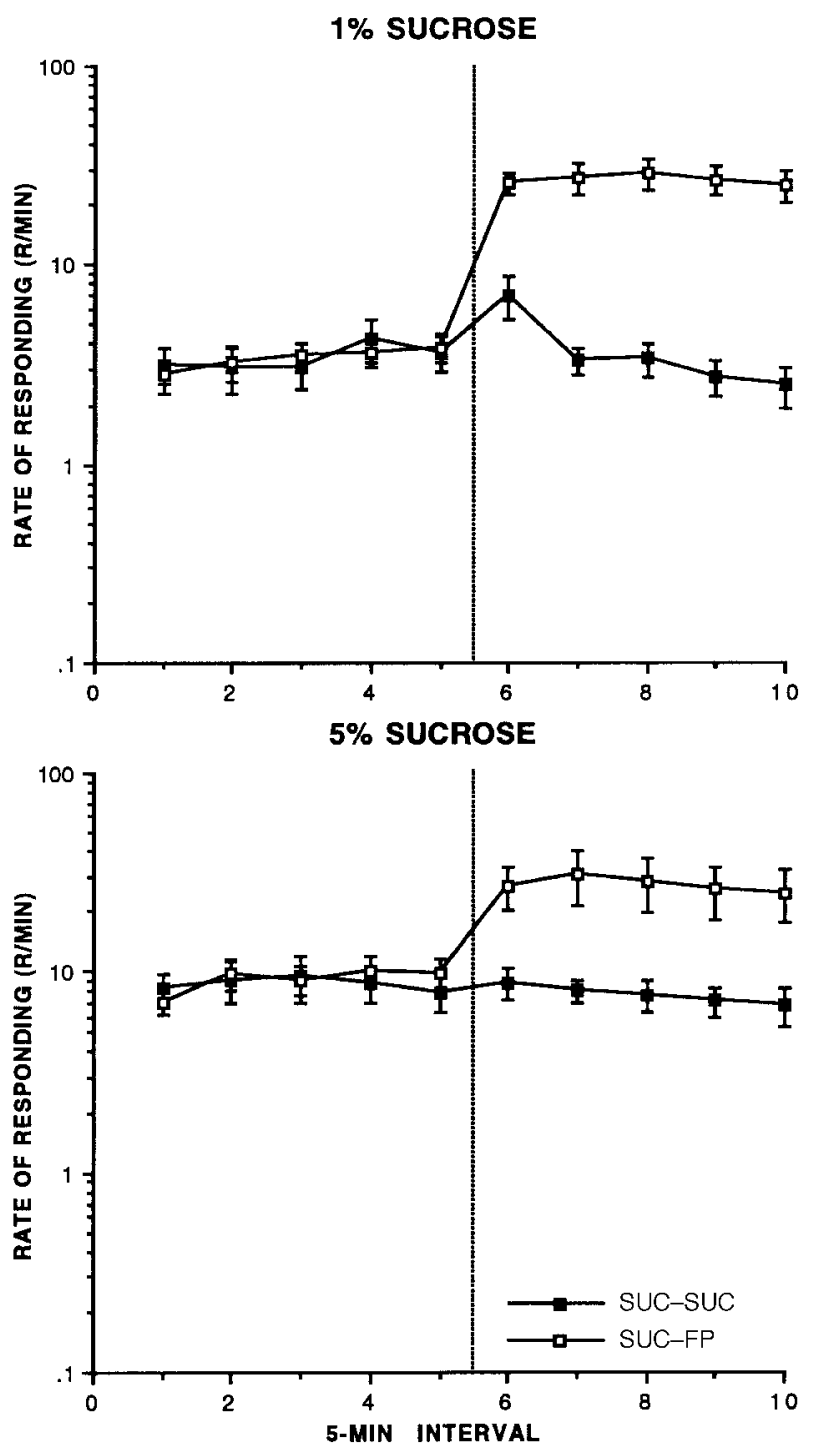

Figure 6. Mean rates of responding for all the subjects as a function of successive 5-min interval in the session for the subjects in the 1\% (top graph) and $5 \%$ (bottom graph) sucrose groups in the sucrose-only (SUC-SUC; closed squares) and sucrose-foodpellet (SUC-FP; open squares) sessions of Experiment 4. Note the logarithmic ordinate.

Figure 7 presents the results from the probe sessions. It was constructed similarly to Figures 4 and 5. Figure 7 provides little evidence that the subjects in either group had a response preference for the lever that had been paired with upcoming food pellet reinforcement. A four-way (group $\times$ reinforcement schedule $\times$ lever $\times 5$-min interval) mixed model ANOVA produced a significant main effect of group $[F(1,10)=5.94]$. The main effect of reinforcement schedule was significant $[F(1,10)=5.83]$, as was the group $\times$ reinforcement schedule interaction $[F(1,10)=15.33]$. The only other effect to reach significance was the group $\times$ lever interaction $[F(1,10)=8.43]$.

Because the interactions between group and reinforcement schedule and between group and lever were signifi- cant, follow-up analyses were conducted separately for the different groups. Responding by the $1 \%$ sucrose group was analyzed by conducting a three-way (reinforcement schedule $\times$ lever $\times 5$-min interval) repeated measures ANOVA. The main effect of lever $[F(1,5)=10.65]$, the main effect of 5-min interval $[F(4,20)=3.21]$, and the three-way interaction $[F(4,20)=4.25]$ were significant. Because of the significant interaction, a two-way (lever $X$ 5 -min interval) repeated measures ANOVA was conducted on responding during the concurrent RI RI sessions. No effects reached significance. An identical analysis was conducted on responding during the extinction sessions. That analysis produced a significant main effect of lever $[F(1,5)=16.08]$ and an interaction between lever and 5-min interval $[F(4,20)=3.04]$. Tests for simple effects indicated that responding on the lever paired with food pellet reinforcement was higher than that on the one paired with sucrose reinforcement during the third 5-min interval $[F(1,5)=16.83]$. However, response rates did not differ during the first, second, fourth, or fifth 5-min intervals.

Responding by the 5\% sucrose group during the probe sessions was also analyzed by conducting a three-way (reinforcement schedule $\times$ lever $\times 5$-min interval) repeated measures ANOVA. The main effect of reinforcement schedule was significant $[F(1,5)=12.58]$. No other effects were significant.

The results from Experiment 4 suggest that when lever location was predictive of upcoming reinforcer type, but not associated with differential overall reinforcement, induction did not result. The results from the probe sessions also provided little evidence for preference for the lever paired with food pellet reinforcement. Only the subjects in the $1 \%$ sucrose group displayed higher response rates on that lever than on the lever paired with upcoming sucrose, and this increase was observed only in the third 5-min interval of the probe sessions in which extinction was in effect.

It could be argued that the failure to observe induction in the present experiment occurred because the subjects failed to discriminate lever location as the predictive stimulus. However, the findings of Experiment 3, in which the subjects responded differentially on the basis of lever location, seemingly indicate that rats are capable of making this discrimination. Furthermore, if the subjects failed to discriminate, they did so despite receiving twice as many training sessions as did the subjects in Experiment 3. In short, although caution should be used when interpreting null results, the present results do not support the idea that Pavlovian processes are behind induction.

\section{GENERAL DISCUSSION}

The present experiments addressed three specific aspects of induction in responding for sucrose produced by upcoming food pellet reinforcement. The first was whether the effect was linked to food pellet reinforcement at all, or whether it occurred because of continued sucrose reinforcement. The second was whether induction occurred because of the conditions of reinforcement in the current 
$1 \%$ SUCROSE CONC RI RI
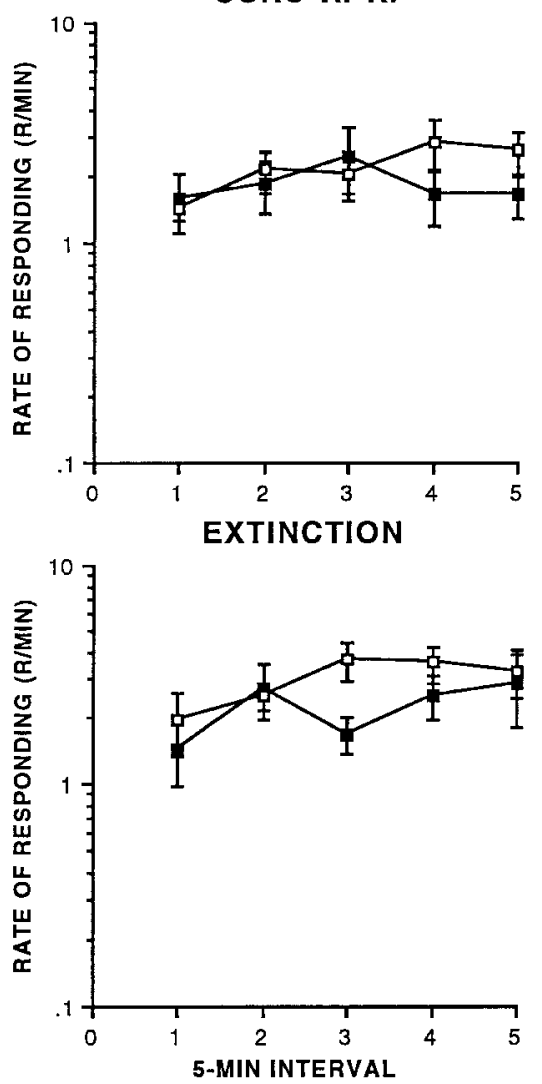

$5 \%$ SUCROSE CONC RI RI
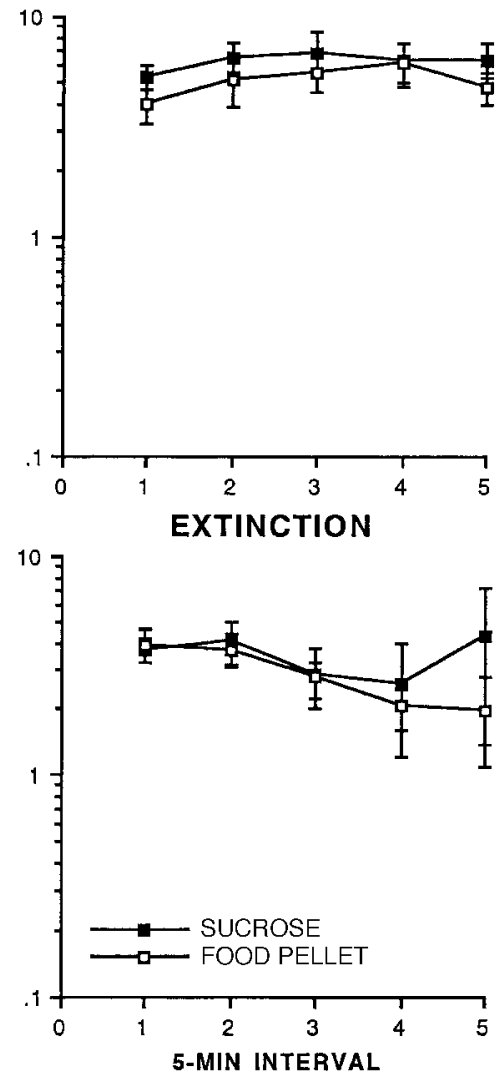

Figure 7. Mean rates of responding in the probe sessions on the lever used in the sucrose-only (closed squares) and sucrose-food-pellet (open squares) sessions for all the subjects in the $1 \%$ (left graphs) and $5 \%$ (right graphs) sucrose groups during sessions in which a concurrent (CONC) random interval RI RI schedule (top graphs) or extinction (bottom graphs) was in effect. Note the logarithmic ordinate.

or in the previous session. The third was whether induction was influenced through Pavlovian processes.

Although previous research (e.g., Weatherly, Davis, \& Melville, 2000; Weatherly, Rue, et al., 2000; Weatherly, Stout, et al., 2000) had characterized the change in responding for sucrose as being produced by upcoming food pellet reinforcement, no conclusive evidence existed to support such a characterization. It remained possible that the continued availability of sucrose reinforcement (in the second half of the session) actually suppressed response rates. Experiment 1 ruled out the latter idea by showing that the subjects responding for sucrose in the first $25 \mathrm{~min}$ of the session responded at a higher rate when food pellets would be available in the second 25 min than when continued sucrose reinforcement, extinction, or nothing (i.e., no second $25 \mathrm{~min}$ ) would be available. Thus, calling the effect induction would appear to be accurate.

Given existing research, it also was not possible to attribute induction to the upcoming food pellets (i.e., prospective influences), because it was possible that it occurred because food pellets had been delivered in the previous session (i.e., retrospective influences). Experiment 2 separated these possibilities by having the subjects respond in sessions in which sucrose reinforcement in the first $25 \mathrm{~min}$ was followed by, in different types of sessions, $25 \mathrm{~min}$ of food pellet or sucrose reinforcement. These session types alternated in strict succession and had specific discriminative stimuli associated with them (i.e., lever location and stimulus lights). After 40 alternating sessions, the subjects responded at a higher rate in the first half of the session when food pellets were upcoming in the second half than when they were not. Because these sessions were always preceded by one in which food pellets had not been available, the results suggest that the induction was controlled by prospective influences.

However, it remained possible that the subjects may have learned that sessions alternated strictly and, thus, increased their rate of response in the first half of the session if food pellets had not been available in the previous session. To investigate this possibility, Experiment 3 replicated the procedure of Experiment 2, with the exception that the different types of session alternated pseudorandomly rather than strictly. Induction was still observed. Because the previous session had little to no predictive 
value for the conditions of reinforcement in the current session, these results further suggest that the induction is prospective rather than retrospective.

In Experiment 3, it was also investigated what stimuli within the current session led to induction. Subsequent to training, the subjects responded in a series of 25-min probe sessions in which both levers were present and either a concurrent RI 60-sec RI 60-sec schedule or extinction was in effect. Across probe sessions, the stimulus lights above the levers were either consistent with or reversed from their position during training. The subjects responded at a higher rate on the lever used in the sucrose-foodpellet sessions than on the one used in the sucrose-only sessions, regardless of where the stimulus lights appeared, indicating that lever location controlled the increased rates of responding.

Experiment 4 explored the role played by lever location. Specifically, it investigated whether, through Pavlovian conditioning, the lever became a conditioned exciter because it had been paired with food pellet reinforcement. The subjects responded in sessions in which lever location in the first half of the session was predictive of the type of reinforcer that would be delivered in the second half. However, responding in the second half of the session was equally likely to occur on either lever. Thus, lever location was predictive of upcoming reinforcement but was equally associated with sucrose and food pellet reinforcement. Induction was not observed, even after 80 training sessions. Furthermore, probe sessions, similar to those described above, showed little difference in responding on the different levers. Thus, the results do not support the idea that Pavlovian processes underlie induction.

These results are consistent with the idea that induction occurs because lever location, in the present procedure, became differentially associated with overall reinforcement. In Experiment 3, when each lever was associated with only one type of session, induction was observed. However, in Experiment 4, when lever location was predictive of the type of upcoming reinforcer but was equally correlated with both sucrose and food pellet reinforcement, it was not. Thus, one could argue that the subjects display induction when in the presence of a stimulus that signals differential reinforcement. One could go further and argue that this process takes place at the level of the entire session. That is, finding induction in Experiment 3, but not in Experiment 4, suggests that the subjects' behavior was determined by the overall reinforcement that would be collected over the entire session.

Such an explanation would appear to be consistent with the present and previous research. In the present study, results between groups responding for $1 \%$ or $5 \%$ sucrose differed little except in the size of the induction effect. This outcome would be predicted because larger relative differences in overall reinforcement would exist between sucrosefood-pellet and sucrose-only sessions when the subjects responded for $1 \%$ sucrose than when they responded for $5 \%$ sucrose. As for previous research, Weatherly, Davis, and Melville (2000) found reductions in induction with reductions in the probability that food pellet reinforcement would occur in the second half of the session. This outcome would be expected because, as the probability of food pellet reinforcement decreased, so too would the level of reinforcement associated with the entire session. Weatherly, Himle, et al. (2001, Experiment 2) found that delaying the upcoming food pellets decreased induction. Inserting an extinction period would lower the relative reinforcing value of the entire session. Weatherly, Himle, et al. (2001, Experiment 3 ) found that induction occurred for responding for sucrose in the first half of the session despite the fact that reinforcement in the first and the second halves were delivered for responding on different levers. If the controlling factor was overall reinforcement at the session level, one would expect induction in this procedure, regardless of which lever delivered the sucrose.

An interesting paradox would arise if the present explanation were accepted. Namely, it would suggest that upcoming food pellet reinforcement is not the key factor. That is, if induction occurs because of the level of reinforcement associated with the entire session, the effect should also be present for responding for sucrose in the second half of the session when food pellets are delivered in the first half. And in fact, both Weatherly, Stout, McMurry, Rue, and Melville (1999) and Weatherly and Moulton (2001) reported finding such a result. However, neither paper established that this increase was an induction effect, because it was possible that the increased response rates occurred, in part, because of thirst brought about by the consumption of the food pellets.

This explanation warrants further exploration. However, it immediately suggests one major concern. If providing food pellet reinforcement in a session increases responding for sucrose by altering overall reinforcement, why does providing sucrose reinforcement in a session in which food pellet reinforcement is also available not decrease response rates for the food pellets, relative to when only food pellet reinforcement will be available (Weatherly \& Moulton, 2001; Weatherly et al., 1999)? Related to this, Weatherly, Stout, Davis, and Melville (2001) did report that responding for $5 \%$ liquid sucrose in the first half of the session decreased when $1 \%$ sucrose (an arguably lower valued reinforcer than $5 \%$ sucrose) would be delivered in the second half versus when 5\% sucrose would be delivered. However, no such effect has been observed for responding for food pellet reinforcement.

The overarching theoretical question that remains is how induction relates to the study of contrast. In reviewing the possible explanations for behavioral contrast, Williams (2002) argued that procedural factors could contribute to, but not explain, contrast. In a similar vein, the present results argue against the idea that induction occurs because of procedural factors (e.g., reinforcement carryover effects from the previous session; Experiments 2 and 3 ). Williams (2002) further argued that steady-state contrast is controlled primarily, if not completely, by the upcoming conditions of reinforcement. The present results also appear to run parallel with this idea in that, for the present procedures, the induction is seemingly controlled by the conditions of reinforcement in the current session. 
Also similar to the present study, Williams (2002; see, also, Williams \& McDevitt, 2001) assessed the potential contribution of Pavlovian conditioning to contrast. He concluded that Pavlovian influences are present within a typical contrast procedure but that they work against the appearance of contrast (perhaps toward the appearance of induction). The results of Experiment 4 do not, however, support the idea that the present induction effect occurs via Pavlovian processes. This dissimilarity leads to several possible conclusions. One is that Pavlovian processes do indeed play a role in the present induction effect but that the procedure of Experiment 4 was unable to elucidate them. Another possibility is that the phenomena of behavioral contrast and induction are distinct and, thus, will not necessarily follow the same causal rules. A third possibility is that both contrast and induction occur through the same causal mechanism (e.g., relative comparisons of the present conditions of reinforcement with those in another situation) but, for reasons yet to be understood, this mechanism leads to induction in some instances and to contrast in others.

As was mentioned above, contrast has interested researchers partially because its appearance is counterintuitive. That is, given the procedures that typically produce it, one would expect to see induction rather than contrast. Williams (2002) implored researchers to pursue an explanation of contrast because "contrast in itself begs for a deeper understanding" (p. 18). He further mused whether perhaps, owing to our failure to date to understand contrast, "like many interesting findings in the conditioning literature, [the study of contrast] may be destined to be added to the historical scrapheap of ideas abandoned prematurely" (p. 18). The present study, for better or worse, helps to showcase our understanding of the relative effects of differential reinforcement. Not only have we yet to understand contrast, we have yet to understand the effect that we would originally expect to observe.

\section{REFERENCES}

Baron, A., \& Derenne, A. (2000). Progressive-ratio schedules: Effects of later schedule requirements on earlier performances. Journal of the Experimental Analysis of Behavior, 73, 291-304.

Catania, A. C. (1973). Self-inhibiting effects of reinforcement. Journal of the Experimental Analysis of Behavior, 19, 517-526.

ElLiotT, M. H. (1928). The effect of change of reward on the maze performance of rats. University of California Publications in Psychology, 4, 19-30.

Flaherty, C. F. (1996). Incentive relativity. Cambridge: Cambridge University Press.

Flaherty, C. F., \& Grigson, P. S. (1988). From contrast to reinforcement: Role of response contingency in anticipatory contrast. Journal of Experimental Psychology: Animal Behavior Processes, 14, 165176.

Flaherty, C. F., \& Rowan, G. A. (1985). Anticipatory contrast: Within-subjects analysis. Animal Learning \& Behavior, 13, 2-5.

Hearst, E., \& Jenkins, H. M. (1974). Sign-tracking: The stimulusreinforcer relation and directed action. Austin, TX: Psychonomic Society.

Hinson, J. J., \& STAdDon, J. E. R. (1978). Behavioral competition: A mechanism for schedule interactions. Science, 202, 432-434.

KilleEN, P. R. (1995). Economics, ecologics, and mechanics: The dynamics of responding under conditions of varying motivation. Journal of the Experimental Analysis of Behavior, 64, 405-431.

McSweeney,F. K., \& Norman, W. D. (1979). Defining behavioral con- trast for multiple schedules. Journal of the Experimental Analysis of Behavior, 32, 457-461.

McSweeney, F. K., \& Weatherly, J. N. (1998). Habituation to the reinforcer may contribute to multiple-schedule behavioral contrast. Journal of the Experimental Analysis of Behavior, 69, 199-221.

MichaEL, J. (1993). Establishing operations. Behavior Analyst, 16, 191206.

Rachlin, H. (1973). Contrast and matching. Psychological Review, 80, 217-234.

REYNoLDS, G. S. (1961). Behavioral contrast. Journal of the Experimental Analysis of Behavior, 4, 57-71.

Timberlake, W., \& ENGLe, M. (1995). Decremental carryover effects of sucrose ingestion in the negative anticipatory contrast procedure in rats. Journal of Experimental Psychology: Animal Behavior Processes, 21, 304-317.

Weatherly, J. N., Davis, C. S., \& Melville, C. L. (2000). Induction with upcoming food-pellet reinforcement. Learning \& Motivation, 31, 180-199.

Weatherly, J. N., Himle, M. B., Plumm, K. M., \& Moulton, P. L. (2001). Three tests of "anticipatory responding" as an account for induction produced by upcoming food-pellet reinforcement. Behavioural Processes, 56, 49-66.

Weatherly, J. N., \& Moulton, P. L. (2001). The effect of food-pellet reinforcement on rats' rates of lever pressing for $1 \%$ sucrose reinforcers across several "contrast" procedures. Learning \& Motivation, 32, 193-218.

Weatherly, J. N., Moulton, P. L., \& Ritt, J. J. (2002). Rats' response rates for $1 \%$ sucrose when food-pellet reinforcement is upcoming: Effect of upcoming reinforcement contingency. Psychological Record, 52, 221-240.

Weatherly, J. N., Rue, H. C., Davis, C. S., \& Melville, C. L. (2000). Delivering different reinforcers in each half of the session: Effect of reinforcement rate. Psychological Record, 50, 543-556.

Weatherly, J. N., Stout, J. E., Davis, C. S., \& Melville, C. L. (2001). For better or worse: Effect of upcoming reinforcer type on rats' lever pressing for low-concentration sucrose reinforcers. Psychological Record, 51, 629-644.

Weatherly, J. N., Stout, J. E., McMurry, A. S., Rue, H. C., \& Melville, C. L. (1999). Within-session responding when different reinforcers are delivered in each half of the session. Behavioural Processes, 46, 227-243.

Weatherly, J. N., Stout, J. E., Rue, H. C., \& Melville, C. L. (2000). The effect of second-half reinforcer type on responding for sucrose in the first half of the session. Behavioural Processes, 49, 43-60.

Williams, B. A. (1983). Another look at contrast in multiple schedules. Journal of the Experimental Analysis of Behavior, 39, 345-384.

Williams, B. A. (2002). Behavioral contrast redux. Animal Learning \& Behavior, 30, 1-20.

Williams, B. A., \& McDevitt, M. A. (2001). Competing sources of stimulus value in anticipatory contrast. Animal Learning \& Behavior, 29, 302-310.

\section{NOTE}

1. Several pieces of evidence support the idea that food pellet reinforcement represents an increase in the conditions of reinforcement over low-concentration sucrose reinforcement. First, food pellets maintain a higher rate of operant responding than does either $1 \%$ or $5 \%$ sucrose (e.g., Weatherly, Davis, \& Melville, 2000; Weatherly, Rue, Davis, \& Melville, 2000; Weatherly, Stout, et al., 2000; the present results). Second, unpublished data from our laboratory indicate that, when both food pellets and sucrose are concurrently available at the same rate, rats allocate more responses (and time) to the food pellet alternative than to the sucrose alternative. Third, a switch from food pellet to $1 \%$ sucrose reinforcement produces negative successive contrast (Elliott, 1928). That is, rats with experience in responding for food pellets respond at a lower rate for $1 \%$ sucrose than do subjects with no experience with food pellet reinforcement (i.e., ones that have always responded for $1 \%$ sucrose; Weatherly \& Moulton, 2001, Experiment 1).

(Manuscript received December 21, 2001; revision accepted for publication August 1, 2002.) 